\title{
A new mutation in the gene encoding mitochondrial seryl-tRNA synthetase as a cause of HUPRA syndrome
}

\author{
Henry Rivera ${ }^{1,2}$, Elena Martín-Hernández ${ }^{3}$, Aitor Delmiro 1,2, María Teresa García-Silva ${ }^{2,3}$, Pilar Quijada-Fraile , \\ Rafael Muley ${ }^{4}$, Joaquín Arenas ${ }^{1,2}$, Miguel A Martín ${ }^{1,2}$ and Francisco Martínez-Azorín ${ }^{1,2^{*}}$
}

\begin{abstract}
Background: HUPRA syndrome is a rare mitochondrial disease characterized by hyperuricemia, pulmonary hypertension, renal failure in infancy and alkalosis. This syndrome was previously described in three patients with a homozygous mutation c.1169A > G (p.D390G) in SARS2, encoding the mitochondrial seryl-tRNA synthetase.

Case presentation: Here we report the clinical and genetic findings in a girl and her brother. Both patients were clinically diagnosed with the HUPRA syndrome. Analysis of the pedigree identified a new homozygous mutation c.1205G > A (p.R402H) in SARS2 gene. This mutation is very rare in the population and it is located at the C-terminal globular domain of the homodimeric enzyme very close to p.D390G.
\end{abstract}

Conclusion: Several data support that p.R402H mutation in SARS2 is a new cause of HUPRA syndrome.

Keywords: Mitochondrial DNA, Mitochondrial disease, HUPRA syndrome, SARS2, Mitochondrial respiratory chain

\section{Background}

Mitochondria are cellular organelles that produce most of the energy that cells use for their functions and survival [1]. Their biogenesis and function is under the genetic control of mitochondrial DNA (mtDNA) and nuclear DNA. Therefore mitochondrial disorders can be originated from mutations in either of the genomes [2]. These disorders represent one of the most common groups of inborn errors of metabolism, with an estimated prevalence of 1 in 5000 births [3]. The kidneys are commonly affected in mitochondrial disease $[4,5]$ because they are aerobic organs with high energy requirements [6].

We report the case of a girl and her brother who presented multiorgan disease, including hyperuricemia, pulmonary hypertension, renal failure, and alkalosis (HUPRA syndrome) (OMIM \#613845) [7]. The sequencing of SARS2

\footnotetext{
* Correspondence: fmartinez@h12o.es

'Laboratorio de Enfermedades Mitocondriales. Instituto de Investigación Hospital 12 de Octubre (i + 12), 6a Planta, Bloque E, Avda. Córdoba s/n, Madrid E-28041, Spain

${ }^{2}$ Centro de Investigación Biomédica en Red de Enfermedades Raras (CIBERER), U723, Madrid E- 28041, Spain

Full list of author information is available at the end of the article
}

gene identified a new homozygous mutation (c.1205G > A p.R402H) as a potential cause of the syndrome.

\section{Case presentation}

\section{Patient II-1}

The patient was a girl born at 37 weeks of gestation of an uneventful pregnancy and delivery. She was the first child of non-consanguineous Spanish parents. At 15 months she was referred to our department because of refractory anemia since the age of 5 months, progressive renal failure with hyperuricemia since the age of 11 months, and metabolic hypochloremic alkalosis despite renal failure. Physical examination was normal except for pallor and mild hypotonia with motor delay. Her weight was $8.250 \mathrm{~kg}$ (percentile 11.5 using WHO reference), her height $76.0 \mathrm{~cm}$ (percentile 30.8) and her cranial perimeter $46 \mathrm{~cm}$ (percentile 61.8). Blood test revealed a normocytic normochromic anemia (Hb $8.4 \mathrm{~g} / \mathrm{dL}(>12)$, VCM 75 fL (70-115), HCM 27 pg (23-35), with normal leucocytes, thrombocytes and reticulocytes), metabolic hypochloremic alkalosis $(\mathrm{pH} 7.51$ (7.35-7.45), $\mathrm{pCO}_{2} 35 \mathrm{mmHg}(27-40), \mathrm{HCO}_{3}^{-} 29.3 \mathrm{mmol} / \mathrm{L}(16-24), \mathrm{Cl}^{-}$ $85 \mathrm{mmol} / \mathrm{L}(95-106))$, renal failure with disproportionately 
higher urea than creatinine (creatinine $1.01 \mathrm{mg} / \mathrm{dL}(0.35-$ $0.5)$, urea, $159 \mathrm{mg} / \mathrm{dL}$ (20-48), estimated creatinine clearance of $42 \mathrm{~mL} / \mathrm{min} / 1.73 \mathrm{~m} 2(>60))$ and hyperuricemia (uric acid $11 \mathrm{mg} / \mathrm{dL}(2.2-7)$ ). Urinalysis displayed a specific gravity of 1.015, pH 5.0, absence of proteinuria and normal cellular sediment. Abdominal ultrasound showed normal kidneys. Her bone marrow aspiration was normal and she had neither ringed sideroblasts nor vacuolization of hematopoietic precursors. Renal and muscle biopsies were performed at the age of 24 months. Renal histology showed normal glomeruli, interstitial fibrosis and tubular atrophy with vacuoles in proximal tubular epithelial cells. Electron microscopy revealed enlarged mitochondria in the cytoplasm of epithelial cells of the proximal tubules. Muscle biopsy demonstrated variations in fiber size, consistent with type 2 fiber atrophy. Cytochrome $\mathrm{C}$ oxidase $(\mathrm{COX})$ and succinate dehydrogenase (SDH) histochemistry were normal. Electron microscopy study of the muscle was normal. Enzymatic activities of mitochondrial respiratory chain in skeletal muscle were normal (Table 1). Deletions of mtDNA were excluded in muscle sample. In additional studies the pancreatic function and the metabolic work up lactic acid, pyruvic acid, amino acids and acylcarnitines were also normal.

In the following months the child required repeated blood transfusions in spite of having received erythropoietin treatment. She presented failure to thrive, hypertension and progressive renal failure. Her overall clinical condition gradually deteriorated and she died at 26 months of multiorgan failure. In the necropsy the only relevant finding was hypertrophic cardiomyopathy.

\section{Patient II-2}

The patient was the younger brother of patient II-1. He was born at 36 weeks of gestational age and had an uneventful neonatal period. At 2 months a normocytic normochromic anemia was found with levels of $\mathrm{Hb}$ of $7.6 \mathrm{~g} / \mathrm{dL}, \mathrm{VCM} 83 \mathrm{fL}, \mathrm{HCM} 28 \mathrm{pg}$, which required repeated blood transfusions since 9 months. Bone marrow was normal even on electron microscopy. At 3 months, a hyperuricemia was found with uric acid levels of $6.6 \mathrm{mg} / \mathrm{dL}$ which increased to $9.4 \mathrm{mg} / \mathrm{dL}$ at the age of 9 months. The fractional excretion of uric acid (FeUA) was constantly low with values of $2-3 \%(>7 \%)$. As a result of failure to thrive since the age of 7 months, continuous nasogastric night feeding and nutritional supplements were administered at the age of 12 months. At 7 months he had hyponatremia with an increased fractional excretion of sodium, and elevated creatinine $(0.96 \mathrm{mg} / \mathrm{dL})$ during a respiratory infection. Similar to his older sister, urea levels were always disproportionately higher than creatinine values and hypochloremic alkalosis was present despite renal insufficiency. Serum magnesium levels were $1.7 \mathrm{mg} / \mathrm{dL}$ (1.5-2.3). Abdominal ultrasound showed normal kidneys. Moreover, lactic acid, amino acids and acylcarnitines in plasma, and organic acids in urine were normal. At 15 months he had normal social and cognitive development with a mild motor delay, his weight was $7.860 \mathrm{~kg}$ (percentile 1.8 using WHO reference), his height $71.5 \mathrm{~cm}$ (percentile 0.1 ) and his cranial perimeter $44 \mathrm{~cm}$ (percentile 1.8). Muscle biopsy was performed and histology, histochemistry and enzymatic studies of respiratory chain were normal (Table 1). Mitochondrial DNA deletions, depletion and frequent mutations were excluded. Other nuclear genes were sequenced with normal results (HNF1, UMOD, CoQ2 and PDSS2). A catheter was placed and ambulatory peritoneal dialysis was started due to creatinine values of $6.3 \mathrm{mg} / \mathrm{dL}$. A renal biopsy was performed at 17 months showing interstitial fibrosis and unspecific tubular damage with normal glomeruli and vascular elements by optic microscopy. Giant and abnormal mitochondria were observed by electron microscopy (Figure 1). Enzyme analysis of respiratory chain complexes in cultured fibroblasts from a skin biopsy at 17 months revealed mild reduced activity of complexes I and IV (Table 1).

At 16 months, severe pulmonary hypertension and right ventricular hypertrophy were diagnosed by echocardiography. In the hemodynamic study, pressures of pulmonary arteries were at the same level as systemic

Table 1 Mitochondrial respiratory chain activities in patients with HUPRA syndrome

\begin{tabular}{|c|c|c|c|c|c|}
\hline \multirow[b]{2}{*}{ Enzyme } & \multicolumn{3}{|c|}{ Skeletal muscle } & \multicolumn{2}{|c|}{ Fibroblasts } \\
\hline & II-1 & $\mathrm{II}-2$ & $C(\text { mean } \pm S D)^{c}$ & $\mathrm{II}-2$ & $C(\text { mean } \pm S D)^{d}$ \\
\hline Citrate synthase (CS) $^{a}$ & 455 & 298 & $550 \pm 350$ & 76 & $87 \pm 13$ \\
\hline Complex I & 14.8 & 19.5 & $15.9 \pm 5.9$ & 13.8 & $19.5 \pm 3.9$ \\
\hline Complex II ${ }^{\mathbf{b}}$ & 14.0 & 11.0 & $12.5 \pm 8.0$ & 41.8 & $39.7 \pm 2.5$ \\
\hline Complex III ${ }^{\mathbf{b}}$ & 73.7 & 34.8 & $68.8 \pm 37.8$ & 63.6 & $63.1 \pm 6.5$ \\
\hline Complex IV & 53.6 & 43.8 & $66.5 \pm 36.5$ & 74.8 & $86.3 \pm 4.2$ \\
\hline
\end{tabular}

In SA (SA is specific activity: $\mathrm{nmol} \times \mathrm{min}^{-1} \times \mathrm{mg}^{\mathrm{a}}$ protein ${ }^{-1}$ ).

${ }^{\mathrm{b}}$ In (SA of Complex / SA of Citrate Synthase) $\times 100$.

'Skeletal muscle from controls: $\mathrm{N}=14$.

${ }^{\mathrm{d}}$ Fibroblasts from controls: $\mathrm{N}=6$.

Bold data indicate that they are below the normal range. 


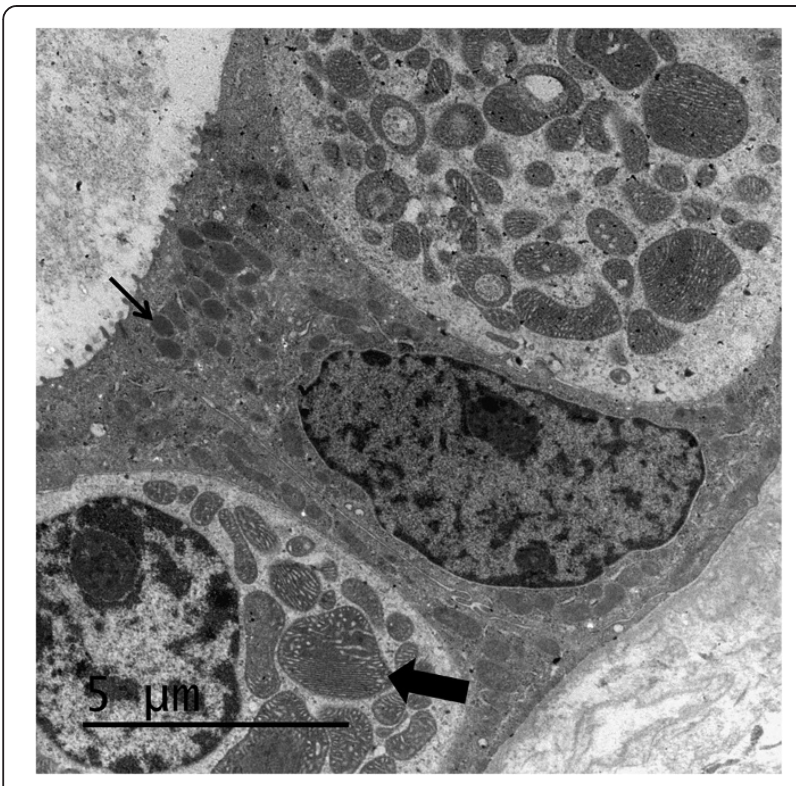

Figure 1 Electron micrograph of a kidney tubule. Abnormal enlarged mitochondria in a portion of the tubular epithelial cells obtained from patient II-2. Normal mitochondrion is marked with a thin arrow and a giant mitochondrion with a wide arrow.

pressures which improved with oxygen and nitric acid administration. Treatment with sildenafil and bosentan showed a partial response. At 21 months he was hospitalized for pneumonia and died of pulmonary hemorrhage, refractory pulmonary hypertension and cardiac failure.

\section{Biochemical studies}

Both patients with HUPRA syndrome were studied for OXPHOS deficiencies in skeletal muscle homogenate [8], and the patient II-2 also in skin fibroblasts [9]. These two methodologies are slightly different being the methodology used in fibroblasts more sensitive and reproducible. The activities of respiratory chain complexes in skeletal muscle homogenate of both patients were normal (Table 1). However, the patient II-2 showed a mild deficiency of complex I and complex IV activities in cultured skin fibroblasts (Table 1), with residual activities being $71 \%$ and $87 \%$ of the mean control values (less than one standard deviation (1SD) away from the mean value).

\section{SARS2 sequencing}

Direct DNA sequencing by Sanger method of all the exons and the adjacent exon-intron boundaries of SARS2 gene [10] was carried out in patient II-2. One homozygous mutation was found (c.1205G > A) resulting in p.R402H. The same missense mutation in a homozygous fashion was detected in patient II-1. And as expected, the parents (I-1 and I-2,) presented the mutation in heterozygosity (Figure 2A and $\mathrm{B}$ ). The aminoacid change affects a highly conserved residue (Figure 2C) and the scores for pathogenicity obtained by in-silico analysis were "damaging" for SIFT [11] and "probably damaging" for PolyPhen-2 [12] software.

\section{Conclusions}

Renal involvement is frequently observed in mitochondrial diseases $[4,5]$, and therefore mitochondrial dysfunction should be considered in the differential diagnosis for unexplained renal disease in infancy $[7,13]$. We have studied two related infants (sister (patient II-1) and brother (patient II-2)) who presented failure to thrive, anemia, metabolic hypochloremic alkalosis, pulmonary hypertension and progressive renal failure with hyperuricemia. They also showed interstitial fibrosis, tubular damage and abnormal mitochondria in kidney. Additionally, one of them also showed a mild deficiency of the mitochondrial respiratory chain in fibroblasts (Table 1). The patients were comprehensively investigated for common etiologies of anemia, uric acid metabolism and mitochondrial metabolism, including sequencing of several genes (HNF1, UMOD, CoQ2, PDSS2 and partially the mtDNA) but no underlying cause was identified. The only reported patients with similar clinical characteristics (3 patients from 2 unrelated Palestinian families from the same village) were diagnosed with the HUPRA syndrome [7] and therefore the patients II-1 and II-2 were clinically diagnosed with this syndrome. The genetic basis of HUPRA syndrome in Palestinian patients was a homozygous mutation (c.1169A > G p.D390G) in the SARS2 gene (RefSeq NG_031865.1) [10], which encodes mitochondrial seryl-tRNA synthetase [7]. The patients II-1 and II-2, unlike the Palestinian patients, had a more severe anemia, a milder developmental delay, normal levels of blood lactate, and normal COX histochemistry and mitochondrial respiratory chain activities in muscle. However, normal respiratory chain activities and morphology in muscle does not rule out the diagnosis of mitochondrial dysfunction. Thus, similar situations have been described before where respiratory chain activities in fibroblasts showed deficiencies while respiratory chain activities and histochemical analysis in muscle were normal [14]. On the other hand, lactic acid elevation in blood is an important, although non-specific and non-sensitive, marker of mitochondrial disease. Many patients with mitochondrial diseases consistently have normal lactic acid levels, even it has been described pathogenic mutations in DARS2 where elevated lactate was only observed in the affected tissue (white matter) and not in blood or cerebrospinal fluid [15]. Additionally, from a cohort of 113 pediatric patients with mitochondrial disease, a significant respiratory chain defect was found in $71 \%$ of the patients, focal absence of COX activity was found only in $13 \%$ of patients and elevation of plasma lactic acid in $60 \%$ of them [16]. Thus, 
A

\section{I}

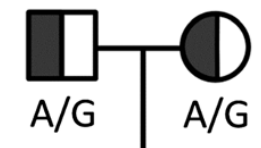

II

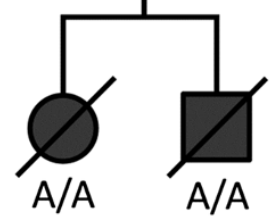

B

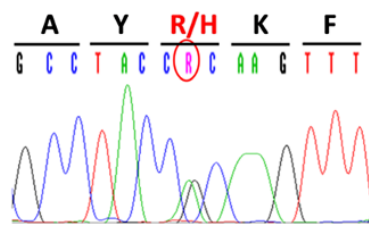

I-1
$\frac{A}{G C \int} \frac{T}{Y} \frac{C}{\int(B) C} \frac{R / H}{A A} \frac{T}{F}$

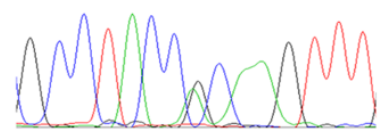

I-2

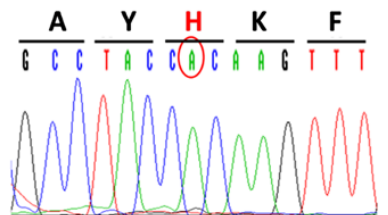

II-1

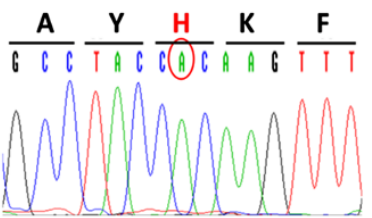

II-2
C

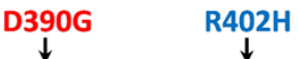

VLDMPTQELGLPAYRKFDIEAWMPGRGRF VLDMPTQELGLPAYRKFDIEAWMPCRGRF VLDMPTQELGLPAYRKFDIEAWMPGRGRF VLDMPTQELGLPTYRKFDIEAWMPGRGHF VLDMPTQELGLPAYRKFDIEAWMPGRGRF VLDMPTQELGLPAYRKFDIEAWMPGRGRY VLDMPTQELGLPAYRKFDIEAWMPGRGRY VLDMPTQELGLPAYRKFDIEAWMPGRGRF VLDMPTQELGLPAYRKFDIEAWMPGRGRF VLDMPTQELGPPAYRKFDIEAWMPGRGSF LLDMPPCELGAPAYRKYDIEAWMPGRAMY TLDMATGDLGAPAYRKFDIEAWMPGLDRY

D TLDMATADLGAPAYRKFDIEAWMPGLGRF
Pan troglodytes

Macaca mulatta

Pongo abelit

Mus musculus

Rattus norvegicus

Canis lupus

Bos taurus

Danio rerio

Anopheles gambiae

Oryza sativa

Arabidopsis thaliana

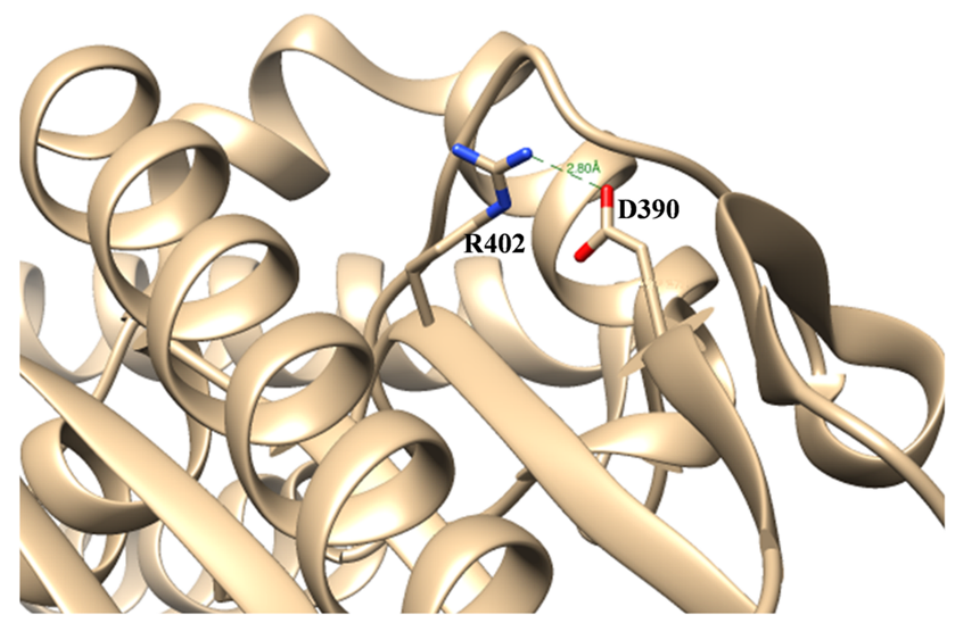

Figure 2 Validation and segregation of c.1205G > A (p.R402H) mutation in SARS2. (A) Family pedigree. (B) Electropherograms showing Sanger sequence validation of the SARS2 c.1205G > A (p.R402H) mutation. (C) Multiple sequence alignment of SARS2 protein region surrounding the novel R402H mutation (blue) in various species. The position of D390G mutation is also indicated (red). (D) Spatial localization of R402 (blue) and D390 (red) is shown in the model of 3D structure of SARS2 protein (PDB code 1WLE). R402 is mutated in patients II-1 and II-2 and D390 is mutated in previously described patients with HUPRA syndrome.

reaching a diagnosis of mitochondrial disease in pediatric patients can be challenging due to it can be accompanied by normal muscle morphology, normal plasma lactate, normal mitochondrial enzymes in skeletal muscle, normal mtDNA mutation screening, and a non-classical clinical presentation.
The description of HUPRA syndrome and it genetic origin due to a mutation in SARS2 gene [7] prompted us to sequence this gene in the patients II-1 and II-2. The sequencing identified a very rare missense mutation in homozygous (c.1205G > A) in both patients that changes a conserved arginine to histidine (p.R402H). The mutation 
is very rare because its allelic frequency is 0.000154 in the population (13006 alleles), and the frequency of homozygous is 0 (in 6503 chromosomes). It has been described only twice in heterozygous in the Exome Sequencing Project (ESP) (https://esp.gs.washington.edu/drupal/). Furthermore, the mutation was predicted to have deleterious effect in the protein by analysis with SIFT [11] and PolyPhen-2 [12] software. It is located in one of the most conserved region of SARS2 and the aminoacid is conserved from Homo sapiens to Arabidopsis thaliana (Figure 2C). The mutation segregates with the disease in this family (Figure 2A and B): the same mutation was detected in patients II-1 and II-2 with identical phenotype, and the parents (I-1 and I-2,) were carriers of the mutation.

SARS2 is a homodimeric enzyme whose active site C-terminal globular domain is built around an eight antiparallel $\beta$-sheet, encompassed by three helical bundles. The aminoacids D390 and R402 are positioned at the C-terminal globular domain, D390 is in the second and R402 in the third $\beta$-strands surrounding an $\alpha$-helix and a $\beta$-turn [17]. In the three-dimensional structure of the protein, both residues are very close $(2.8 \AA)$ (Figure 2D) [18], which could explain why mutations in these residues give rise to similar clinical phenotypes.

Recently, mutations in genes encoding mitochondrial aminoacyl-tRNA synthetases (mtARSs) have emerged as a new cause of human disease, resulting in surprisingly tissue-specific phenotypes, although they are all expected to impair mitochondrial protein synthesis and thus affect the OXPHOS system. However, the molecular mechanisms behind the selective tissue involvement are not currently understood. Several possibilities have been pointed out to explain the tissue-specificity phenotype of mtARS mutation [19]: (i) the remaining residual activity of mtARS is sufficient to maintain mitochondrial translation in most cell types but not in the specific tissues; (ii) tissue-specific differences in mitochondrial chaperone activities may play a role in determining the stability of some mutants; (iii) tissue-specific levels of particular uncharged tRNAs and amino acids; and (iv) mtARS could also have additional functions that are indispensable in specific cell types or developmental stages.

The principal affected tissue in HUPRA syndrome by SARS2 mutations is the kidney. Mutations in SARS2 resulting in decreased aminoacylation of one tRNA isoacceptor ( $t R N A{ }_{\text {AGY }}^{\mathrm{Ser}}$ or $\mathrm{tRNA}^{\mathrm{Ser}}{ }_{\mathrm{UCN}}$ ) by the serine amino acid are expected to adversely affect mitochondrial translation systems and lead to derangements in the synthesis of mitochondrial proteins and consequently in energy supply. Reduced energy production may account for impaired tubular function, which is known to be especially vulnerable to mitochondrial dysfunction since it has very high energy requirements [6].
In conclusion, there are several findings that support the pathogenic role of $\mathrm{R} 402 \mathrm{H}$ change: (i) the mutation is very rare in the population; (ii) the substitution is predicted to have deleterious effect in the protein; (iii) the aminoacid residue is highly conserved (Figure 2C); (iv) the mutation segregates with the disease (Figure 2A); (v) the patient fibroblasts have a combined deficiency in complexes of the mitochondrial respiratory chain (Table 1) as expected for defects in a gene involved in mitochondrial protein synthesis; and (vi) the mutation is located close to the only described mutation in SARS2 associated with HUPRA syndrome (Figure 2D) [7]. Finally, we consider that the SARS2 gene must be the first line of investigation when the HUPRA phenotype is present.

\section{Consent}

Informed written consent was obtained from the patient's parents, and the Ethic Committee of the Instituto de Investigación Hospital 12 de Octubre $(\mathrm{i}+12)$ approved the study.

\section{Abbreviations}

HUPRA: Hyperuricemia, pulmonary hypertension, renal failure, and alkalosis; SARS2: Mitochōndrial seryl-tRNA synthetase; mtDNA: Mitochondrial DNA; ESP: Exome Sequencing Project; WHO: World Health Organization.

\section{Competing interests}

The authors have declared that no competing interests exist.

\section{Authors' contributions}

HR carried out molecular genetic studies and helped to draft the manuscript. EMH, MTGS, PQF, RM and JA participated in clinical evaluation. AD carried out enzymatic and in silico studies. MAM helped to draft the manuscript. FMA conceived and designed the experiments, analyzed the data and drafted the manuscript. All authors read and approved the final manuscript.

\section{Acknowledgements}

We would like to thank Alicia Torrado for valuable comments on the manuscript and Aida Isabel Molero Bermejo for her assistance in the analysis of electron micrographs of kidney. This work was supported by grants from the Spanish Ministerio de Economía y Competitividad (ISCIII CP08/00018 and PI10/00063 to FMA; and ISCIII PI12/01683 to MAM).

\section{Author details}

${ }^{1}$ Laboratorio de Enfermedades Mitocondriales. Instituto de Investigación Hospital 12 de Octubre $(i+12)$, $6^{a}$ Planta, Bloque E, Avda. Córdoba s/n, Madrid E-28041, Spain. ${ }^{2}$ Centro de Investigación Biomédica en Red de Enfermedades Raras (CIBERER), U723, Madrid E- 28041, Spain. ${ }^{3}$ Unidad Pediátrica de Enfermedades Raras, Hospital 12 de Octubre, Madrid E-28041, Spain. ${ }^{4}$ Unidad Pediátrica de Nefrología, Hospital 12 de Octubre, Madrid E-28041, Spain

Received: 7 May 2013 Accepted: 4 September 2013 Published: 13 September 2013

\section{References}

1. Attardi G, Schatz G: Biogenesis of mitochondria. Annu Rev Cell Biol 1988 4:289-333

2. Tucker EJ, Compton AG, Thorburn DR: Recent advances in the genetics of mitochondrial encephalopathies. Curr Neurol Neurosci Rep 2010, 10(4):277-285

3. Skladal D, Halliday J, Thorburn DR: Minimum birth prevalence of mitochondrial respiratory chain disorders in children. Brain 2003, 126(Pt 8):1905-1912 
4. Rahman S, Hall AM: Mitochondrial disease--an important cause of endstage renal failure. Pediatr Nephrol 2013, 28(3):357-361.

5. Martin-Hernandez E, Garcia-Silva MT, Vara J, Campos Y, Cabello A, Muley R, Del Hoyo P, Martin MA, Arenas J: Renal pathology in children with mitochondrial diseases. Pediatr Nephrol 2005, 20(9):1299-1305.

6. Bagnasco S, Good D, Balaban R, Burg M: Lactate production in isolated segments of the rat nephron. Am J Physiol 1985, 248(4 Pt 2):F522-526.

7. Belostotsky R, Ben-Shalom E, Rinat C, Becker-Cohen R, Feinstein S, Zeligson S, Segel R, Elpeleg O, Nassar S, Frishberg Y: Mutations in the mitochondrial seryl-tRNA synthetase cause hyperuricemia, pulmonary hypertension, renal failure in infancy and alkalosis, HUPRA syndrome. Am J Hum Genet 2011, 88(2):193-200.

8. Martinez B, del Hoyo P, Martin MA, Arenas J, Perez-Castillo A, Santos A: Thyroid hormone regulates oxidative phosphorylation in the cerebral cortex and striatum of neonatal rats. J Neurochem 2001, 78(5):1054-1063.

9. Medja F, Allouche S, Frachon P, Jardel C, Malgat M, Mousson de Camaret B, Slama A, Lunardi J, Mazat JP, Lombes A: Development and implementation of standardized respiratory chain spectrophotometric assays for clinical diagnosis. Mitochondrion 2009, 9(5):331-339.

10. Yokogawa T, Shimada N, Takeuchi N, Benkowski L, Suzuki T, Omori A, Ueda T, Nishikawa K, Spremulli LL, Watanabe K: Characterization and tRNA recognition of mammalian mitochondrial seryl-tRNA synthetase. J Biol Chem 2000, 275(26):19913-19920.

11. Kumar P, Henikoff S, Ng PC: Predicting the effects of coding nonsynonymous variants on protein function using the SIFT algorithm. Nat Protoc 2009, 4(7):1073-1081.

12. Adzhubei IA, Schmidt S, Peshkin L, Ramensky VE, Gerasimova A, Bork P, Kondrashov AS, Sunyaev SR: A method and server for predicting damaging missense mutations. Nat Methods 2010, 7(4):248-249.

13. D'Aco KE, Manno M, Clarke C, Ganesh J, Meyers KE, Sondheimer N: Mitochondrial tRNA(Phe) mutation as a cause of end-stage renal disease in childhood. Pediatr Nephrol 2013, 28(3):515-519.

14. Oglesbee D, Freedenberg D, Kramer KA, Anderson BD, Hahn SH: Normal muscle respiratory chain enzymes can complicate mitochondrial disease diagnosis. Pediatr Neurol 2006, 35(4):289-292.

15. Scheper GC, van der Klok T, van Andel RJ, van Berkel CG, Sissler M, Smet J, Muravina TI, Serkov SV, Uziel G, Bugiani M, et al: Mitochondrial aspartyltRNA synthetase deficiency causes leukoencephalopathy with brain stem and spinal cord involvement and lactate elevation. Nat Genet 2007, 39(4):534-539.

16. Scaglia F, Towbin JA, Craigen WJ, Belmont JW, Smith EO, Neish SR, Ware SM, Hunter JV, Fernbach SD, Vladutiu GD, et al: Clinical spectrum, morbidity, and mortality in 113 pediatric patients with mitochondrial disease. Pediatrics 2004, 114(4):925-931.

17. Chimnaronk S, Gravers Jeppesen M, Suzuki T, Nyborg J, Watanabe K: Dualmode recognition of noncanonical tRNAs(Ser) by seryl-tRNA synthetase in mammalian mitochondria. EMBO J 2005, 24(19):3369-3379.

18. Pettersen EF, Goddard TD, Huang CC, Couch GS, Greenblatt DM, Meng EC, Ferrin TE: UCSF Chimera-a visualization system for exploratory research and analysis. J Comput Chem 2004, 25(13):1605-1612.

19. Konovalova S, Tyynismaa $\mathrm{H}$ : Mitochondrial aminoacyl-tRNA synthetases in human disease. Mol Genet Metab 2013, 108(4):206-211.

\section{doi:10.1186/1471-2369-14-195}

Cite this article as: Rivera et al: A new mutation in the gene encoding mitochondrial seryl-tRNA synthetase as a cause of HUPRA syndrome. BMC Nephrology 2013 14:195.

\section{Submit your next manuscript to BioMed Central and take full advantage of:}

- Convenient online submission

- Thorough peer review

- No space constraints or color figure charges

- Immediate publication on acceptance

- Inclusion in PubMed, CAS, Scopus and Google Scholar

- Research which is freely available for redistribution 